\title{
AMBULATÓRIO DE QUIMIOTERAPIA PEDIÁTRICA: A EXPERIÊNCIA NO AQUÁRIO CARIOCA ${ }^{1}$
}

\author{
Isabelle Pimentel Gomes ${ }^{2}$, Neusa Collet ${ }^{3}$, Paula Elaine Diniz dos Reis ${ }^{4}$
}

\begin{abstract}
${ }^{1}$ Pesquisa financiada pela Fundação para o Desenvolvimento Científico e Tecnológico em Saúde (FIOTEC), Fundação Oswaldo Cruz.

${ }^{2}$ Doutoranda do Programa de Pós-Graduação em Enfermagem da Universidade Federal da Paraíba (UFPB). Enfermeira da Clínica Pediátrica do Hospital Universitário Lauro Wanderley. Bolsista FIOTEC. Paraíba, Brasil. E-mail: enfisabelle@yahoo. com.br

${ }^{3}$ Doutora em Enfermagem. Professora do Departamento de Enfermagem em Saúde Pública e Psiquiatria do Centro de Ciências da Saúde e do Programa de Pós-Graduação em Enfermagem da UFPB. Paraíba, Brasil. E-mail: neucollet@gmail.com

${ }^{4}$ Doutora em Enfermagem. Professora Adjunto do Departamento de Enfermagem da Universidade de Brasília. Brasília, Brasil. E-mail: pauladiniz@unb.br
\end{abstract}

RESUMO: Objetiva-se relatar a experiência, enquanto enfermeira, de um ambulatório de quimioterapia pediátrica, que foi decorado para ser atraente às crianças (Aquário Carioca). Trata-se de um estudo descritivo do tipo relato de experiência, em um hospital do Rio de Janeiro, realizado entre dezembro de 2007 e maio de 2008. Este ambiente foi decorado baseado no filme Procurando Nemo (Disney$\left.\operatorname{Pixar}^{\circledR}\right)$, após um acordo firmado entre a instituição e representantes da sociedade civil. O Aquário Carioca trouxe o brincar para o local de tratamento. Percebeu-se que os profissionais pareciam mais motivados, o cuidado foi facilitado e as relações tornaram-se mais dialógicas ao utilizar recursos lúdicos, minimização da agitação da criança durante procedimentos, diminuição de náuseas e vômitos, desejo da criança em permanecer na sala. A mudança do ambiente ofereceu às crianças atividades estimulantes e divertidas, trazendo distração, calma, segurança e maior aceitação do tratamento. Com isso a criança desviou seu foco da doença.

DESCRITORES: Ambiente de instituições de saúde. Enfermagem oncológica. Enfermagem pediátrica. Humanização da assistência. Quimioterapia.

\section{PEDIATRIC OUTPATIENT CHEMOTHERAPY: EXPERIENCE IN THE CARIOCA AQUARIUM}

\begin{abstract}
The objective of this study was to report the nursing experience at a pediatric chemotherapy outpatient clinic that was decorated to be appealing to children (Carioca Aquarium). It is a descriptive experience report from a hospital in Rio de Janeiro, Brazil, conducted between December, 2007, and May, 2008. The environment was decorated based on the (Disney-Pixar ${ }^{\circledR}$ ) movie, "Finding Nemo" after an agreement between the institution and community representatives. Carioca Aquarium brought the act of playing to the treatment site. We observed that professionals were more motivated, care was facilitated, and relationships involved more dialogue when using recreational resources. In turn, children's agitation during procedures was minimized, nausea and vomiting was reduced, and the children's desire to remain in the room increased. The change of environment offered stimulating and fun activities to the children, bringing them distraction, calmness, security, and greater acceptance of their treatment. With this, the children were able to divert their focus from their disease.
\end{abstract}

DESCRIPTORS: Health facility environment. Oncologic nursing. Pediatric nursing. Humanization of Assistance. Drug therapy.

\section{QUIMIOTERAPIA PEDIÁTRICA: EXPERIENCIA EN EL ACUARIO CARIOCA}

RESUMEN: El objetivo del estudio es relatar la experiencia como enfermera en una quimioterapia pediátrica, decorada para ser atractiva
para los niños (Acuario Carioca). Se trata de un estudio de carácter descriptivo con relato de la experiencia vivida en un hospital de la
ciudad de Rio de Janeiro,Brazil, entre diciembre de 2007 a mayo de 2008. La decoración del espacio se basó en la película "Buscando
a Nemo" (Disney-Pixar ${ }^{\circledR}$ ), después de un acuerdo entre la institución y la sociedad civil. El Acuario Carioca llevó la diversión al lugar
de tratamiento. Se observó que los profesionales parecían más motivados, se facilitó la atención y las relaciones se han vuelto más
dialógicas cuando se utilizan los recursos recreativos, reduciendo la agitación de los niños durante los procedimientos, reducción de
náuseas y vómitos, deseo de los niños a permanecer en la sala. El cambio del espacio ofrece actividades estimulantes y divertidas,
distracción, calma, seguridad y una mayor aceptación del tratamiento. Así, el niño cambió su enfoque de la enfermedad. DESCRIPTORES: Ambiente de instituciones de salud. Enfermería oncológica. Enfermería pediátrica. Humanización de la Atención. Quimioterapia. 


\section{INTRODUÇÃO}

O diagnóstico de câncer infantil promove rápida e intensa transformação na vida da criança e no cotidiano familiar. De um momento para o outro, eles se veem em um hospital, um ambiente estranho, muitas vezes nada hospitaleiro, com pessoas desconhecidas, no qual a criança será submetida a exames e tratamentos invasivos e dolorosos, sendo afastada de seu ambiente, familiares e amigos. Independente da idade da criança e de sua capacidade de compreensão cognitiva da realidade que a rodeia, ela, de algum modo, percebe que algo grave e temível está acontecendo consigo. Frente a esta realidade a criança pode ter seu desenvolvimento subitamente modificado por vivenciar sensação de perigo iminente. ${ }^{1}$

Sob esta ótica, a hospitalização exerce influência negativa na vida das crianças, muitas vezes traumatizante, e pode ser ainda pior, quando a permanência no ambiente é necessária em decorrência do diagnóstico de câncer, cujo tratamento é prolongado, agressivo e doloroso. O tratamento de doenças crônicas geralmente é longo, complexo, demandando cuidados constantes em relação à terapêutica em si e em relação a determinantes que possam agravar o estado de saúde da criança. ${ }^{2}$

A quimioterapia ambulatorial surge como uma alternativa para minimizar danos relacionados a quebras de vínculos familiares decorrentes da hospitalização, uma vez que a criança tem possibilidade de receber o tratamento proposto e ser liberada para retornar ao lar após algumas horas. Alguns tipos de câncer infantil são tratados exclusivamente de forma ambulatorial, sendo a internação hospitalar somente utilizada para atender possíveis complicações. Contudo, muitas dificuldades precisam ser enfrentadas pela criança e família, independente do tratamento ser em regime ambulatorial ou em internação, estando relacionadas ao processo do adoecer e da necessidade de idas frequentes ao hospital. ${ }^{3}$

É comum identificar no cotidiano hospitalar crianças sendo assistidas com qualidade técnica e de alta complexidade, sendo tratadas adequadamente sob a ótica clínica e, ao mesmo tempo, vivenciando situações desconfortáveis, nada humanizadas, podendo promover estresse à criança e à sua família, interferindo na recuperação da saúde da criança e levando ao agravamento do quadro instalado.
Diante desta realidade, a humanização hospitalar torna-se premente. O ser humano deve ser visto em sua condição simultânea, complexa, exuberante e problemática, sobretudo em relação aos processos saúde/doença. ${ }^{4}$ Se este ser humano é uma criança suas singularidades devem ser valorizadas, mesmo que o seu mundo seja diferente do mundo dos profissionais e dos gestores. Recentemente, pesquisas tem salientado a influência que o espaço físico tem sobre os diferentes atores no ambiente hospitalar, tais como os pacientes, $\mathrm{o}$ corpo técnico e os acompanhantes. ${ }^{5-6}$

O Ministério da Saúde (MS) vem buscando qualificar os serviços de saúde, visando os usuários do sistema como ser humano com aspectos físicos, sociais e psicológicos que precisam ser respeitados, portanto intencionando melhorar a qualidade dos hospitais públicos que integram a rede do Sistema Único de Saúde (SUS).?

Dentre outras diretrizes, o MS enfatiza a humanização como política que transversaliza todo sistema: das rotinas nos serviços às instâncias e estratégias de gestão, criando operações capazes de fomentar trocas solidárias, em redes multiprofissionais e interdisciplinares; implicando gestores, profissionais e usuários em processos humanizados de produção dos serviços, a partir de novas formas de pensar e cuidar da saúde, e de enfrentar seus agravos. As diretrizes requerem que haja apropriação dos processos de trabalho, por gestores, profissionais e usuários, com base em uma ética de coimplicação na produção dos saberes, das práticas e das relações no campo da saúde, por meio da comunicação, colaboração e compartilhamento entre esses atores, nas diferentes ações e instâncias gestoras do SUS. ${ }^{4}$

Tendo em vista a humanização, o conceito de ambiente hospitalar foi ampliado e passou a ser entendido como ecologia hospitalar, englobando as dimensões das relações, a estrutura física e, especialmente, o modo como estas duas interagem com as atividades que ali ocorrem, com as histórias ali narradas, com as pessoas que por elas transitam. ${ }^{8}$ A estrutura física deve ser entendida não apenas em sua conformação de estruturas não humanas, formas, cores, texturas, organização, proporções e símbolos, pois nela e com ela se estabelecem laços afetivos. A ecologia hospitalar envolve aspectos específicos à infância que estão relacionados às condições biológicas, necessárias para realização de tratamento; sociais, que se dão pela possibilidade do brincar e se relacionar com outras crianças, profissionais e familiares; psicológicas, que 
reduzam o medo e o sofrimento decorrentes dos procedimentos e toxicidades quimioterápicas, trazendo conforto e bem-estar. ${ }^{9}$

As características do processo de humanização visadas pela Política Nacional de Humanização (PNH), HumanizaSUS, vêm ao encontro da ecologia hospitalar, pois reconhece esse ambiente composto por todos os processos que permeiam o cuidado de saúde em seu espaço físico. Sabe-se que este exerce forte influência sobre o desenvolvimento do cuidado para os profissionais, bem como para a criança e sua família, merecendo destaque.

Transformar o ambiente hospitalar que faz atendimento a crianças em um ambiente lúdico, atraente, com a incorporação da fantasia, trazendo uma aproximação a parques infantis é um desafio para o sistema público de saúde devido à elevação dos custos iniciais e de manutenção. ${ }^{6}$ Entretanto, já existem algumas instituições que alcançaram tal desafio. Como, no geral, esta não é a realidade dos hospitais públicos brasileiros, torna-se relevante divulgar as experiências vivenciadas em locais que se destacam por buscar a humanização do cuidado. Assim, neste estudo traçou-se como objetivo relatar a experiência enquanto enfermeira de um ambulatório de quimioterapia pediátrica que foi decorado para ser atraente às crianças (Aquário Carioca).

\section{METODOLOGIA}

Trata-se de um estudo descritivo do tipo relato de experiência, da atuação, enquanto enfermeira em ambulatório de quimioterapia pediátrica com ambiente humanizado, em um hospital público na cidade do Rio de Janeiro-RJ, realizado no período de dezembro de 2007 a maio de 2008. Para a descrição da experiência foram considerados os seguintes aspectos: abordar as mudanças ocorridas no espaço físico da unidade de atendimento ambulatorial a crianças em quimioterapia e sua importância para a humanização do cuidado no cotidiano da atenção de enfermagem com vistas aos preceitos da ecologia hospitalar.

Por caracterizarem-se como narrativas de experiência profissional, os relatos de experiência buscam construir "conhecimentos vindos do cotidiano, alicerçados em bibliografias que as referendam". ${ }^{10: 117}$ Neste estudo foram seguidos os seguintes passos metodológicos: esclarecimento da situação inicial; explicitação dos componentes dos fatos apresentando bibliografias de diferen- tes fontes; detalhamento das informações sobre a experiência; apresentação da sequência dos acontecimentos mesclados de opiniões e sugestões denotando as evoluções e mudanças ocorridas no contexto. $^{10}$

O estudo foi desenvolvido atendendo aos aspectos éticos conforme a Resolução 196/96 do Conselho Nacional de Saúde, ${ }^{11}$ e foi submetido à apreciação do Comitê de Ética em Pesquisa tendo recebido parecer favorável (Protocolo $n^{0} 56 / 09$ ).

\section{CONHECENDO COMO TUDO COMEÇOU}

O hospital em estudo realiza atendimento à criança com câncer. Nesta Instituição, até dezembro de 2007, a quimioterapia era realizada em uma sala pequena, que não proporcionava conforto às crianças, família e profissionais, com poucas opções de brinquedos e distração. Devido à restrição de espaço, tornava-se inviável oferecer opções lúdicas no ambiente, pois a sala era ocupada com materiais e equipamentos fundamentais para intervenções à criança durante a quimioterapia.

As condições físicas precárias têm impacto sobre a ação profissional e o conforto das crianças. Ao mesmo tempo, essas condições não se configuram como impeditivos para a construção de um ambiente onde ações ou "aspectos lúdicos" podem ser identificados. ${ }^{12}$ Mesmo com as condições relatadas, a instituição desenvolvia projetos de humanização que beneficiavam as crianças com câncer, porém, apenas um projeto era realizado no ambulatório de quimioterapia: a visita dos Doutores da Alegria.

Tendo em vista a necessidade de adequação do espaço físico para uma nova proposta de produção do cuidado que visava o alcance das diretrizes do HumanizaSUS, destinou-se uma nova área para a sala de quimioterapia, a qual foi submetida à ampla reforma. Criou-se uma parceria da Instituição com a sociedade civil, representada neste caso pelo cenógrafo Gringo Cardia e o Instituto Desiderata, o qual possui dentre os objetivos contribuir para o processo de transformação e inclusão social de crianças e jovens, na área de Oncologia Pediátrica. ${ }^{13}$

A parceria resultou na decoração do ambiente da sala de quimioterapia com base no filme Procurando Nemo (Disney-Pixar $\left.{ }^{\circledR}\right)$, sendo denominada Aquário Carioca, transformando o ambiente no mundo mágico do fundo do mar. 


\section{O AQUÁRIO CARIOCA}

O Aquário Carioca foi criado objetivando oferecer espaço acolhedor para as crianças, seus familiares e os profissionais, integrando ao tratamento a oportunidade de desenvolvimento e expressão de todos. Foi inaugurado no dia 07 de dezembro de 2007, ${ }^{13}$ com um cenário magnífico, que lembra os parques da Disney ${ }^{\circledR}$.

O Aquário Carioca é um local amplo, composto por: recepção, decorada como praia; consultório, colorido com verde; sala de quimioterapia, decorada como fundo do mar; sala de procedimentos invasivos e emergência, colorida com azul; posto de enfermagem, o qual segue decoração do fundo do mar; banheiro, específico para crianças; toalete; expurgo e depósito de material limpo.

Este local tinha capacidade para atender sete crianças em tratamento concomitantemente, tendo cinco poltronas e duas camas, disponibilizadas de forma a oportunizar privacidade, caso fosse uma necessidade da criança. A permanência do familiar ao lado da criança durante a quimioterapia era incentivada, sendo-lhes oferecido cadeiras individuais e confortáveis. Além disso, encontravam-se à disposição das crianças, opções lúdicas (brinquedos, móbiles, livros, cadernos de desenhos, videogame, som ambiente, televisões com vídeos infantis, entre outros) que podiam ser utilizados durante a espera para atendimento e infusão da quimioterapia.

$\mathrm{Na}$ inauguração deste espaço, a alegria da equipe multiprofissional pela realização de um sonho foi emocionante. A surpresa e o encantamento das crianças ao entrarem no Aquário Carioca foram comoventes. Esse efeito positivo não ocorreu só no dia da inauguração, continuou perdurando. A partir do Aquário Carioca foi possível presenciar momentos muito especiais da influência deste ambiente no tratamento das crianças sobre os diferentes aspectos que envolvem a ecologia hospitalar, biológicos, sociais e psicológicos, como por exemplo, a superação de um menino com 10 anos que apresentava náuseas e vômitos antecipatórios. Esses tipos de náuseas e vômitos são causados por um reflexo condicionado, sendo comuns na véspera da sessão de quimioterapia, ou mesmo, podem ocorrer por uma simples visão do hospital, gerando um efeito nauseante forte. ${ }^{14}$ No seu primeiro dia de infusão no novo espaço, ele chegou ao setor apresentando tais náuseas, foi puncionada uma veia periférica, e a medicação foi infundida com as doses e diluições padronizadas. Era comum esta criança apresentar episódios de vômitos durante a administração da quimioterapia, porém, neste dia, desfrutando do Aquário Carioca, e jogando vídeo-game durante todo o período da infusão, não apresentou vômito. A equipe de enfermagem e a mãe se alegraram, pois nenhuma medicação antiemética utilizada nos ciclos anteriores tinha apresentado tal resultado. Com isso, ele teve diminuição dos sintomas e evitou o constrangimento que frequentemente era observado na criança, quando da ocorrência dos vômitos, uma vez que ficava inibido frente às outras crianças que recebiam quimioterapia. A satisfação da criança e a alegria da mãe ao final do ciclo foram contagiantes.

Pesquisas sugerem que o uso de intervenções não farmacológicas para náuseas e vômitos induzidos por quimioterapia deva ser considerado em pacientes com câncer, principalmente naqueles que experimentam recorrência de êmese em ciclos subsequentes. ${ }^{15}$ Dentre as intervenções encontra-se a técnica de relaxamento. No caso descrito, o vídeo-game pode ter provocado relaxamento, levando a criança a tirar o foco do tratamento, mantendo-se concentrado no jogo. Assim, os aspectos biológicos ${ }^{8}$, representados neste caso pela redução de sintomas e psicossociais, ${ }^{8}$ pela redução do constrangimento e do desconforto gerado pelos vômitos, sofreram influência do ambiente do Aquário Carioca, com presença do lúdico, concomitante ao tratamento, que contribuiu para a satisfação dos envolvidos no cuidado. Com isso, o ambiente do tratamento pode ser relacionado ao lazer, deixando de ser apreendido apenas como um local de procedimentos que gera sofrimento.

Outro menino, com aproximadamente quatro anos de idade, apresentava difícil acesso venoso periférico e, por estar no fim do tratamento endovenoso, a opção foi por não colocar cateter venoso central. Quando ele era atendido na antiga sala de quimioterapia, apresentava-se estressado, agitado, agressivo, irritado e choroso durante as tentativas de punção venosa. Na primeira vez que foi atendido no Aquário Carioca, a criança foi colocada na maca para puncionar a veia periférica e, no momento, tocava uma música de sons da natureza, ouvia-se o cantar de gaivotas, o barulho do vento e os sons de ondas do mar. O menino ficou procurando onde estavam os passarinhos que cantavam e ficou sossegado, tranquilo e relaxado, permitindo um momento 
mais calmo e seguro, possibilitando a punção venosa com facilidade. Por fim, ele dormiu, sem a agitação que comumente acontecia. $\mathrm{O}$ som não só tranquilizou o menino, como também ajudou a enfermagem a conhecer mais uma opção para distraí-lo, fazendo com que destinasse sua atenção ao ambiente e não ao procedimento invasivo, agressivo, doloroso e estressante também para a equipe. O som proporcionou alívio das tensões da criança, mãe e equipe.

Uma revisão integrativa ${ }^{16}$ verificou que entre os resultados positivos obtidos com a intervenção musical, estavam: redução e controle da dor e de comportamentos causados por ela; diminuição da agitação e de comportamentos agressivos; redução da ansiedade; relaxamento; diminuição do medo e sofrimento; melhora nas habilidades cognitivas, sociais e físicas; indução do sono; modulação do humor; distração/divertimento; reabilitação e satisfação do cliente e familiares com o cuidado prestado. A música é um recurso eficaz para qualificar o cuidado à criança, principalmente quando está diante de um procedimento invasivo e doloroso. Além disso, a técnica de distração é frequentemente utilizada como uma intervenção não farmacológica para a dor. ${ }^{17}$

Estes fatos descritos corroboram as expectativas de que a mudança no ambiente e as estratégias lúdicas de humanização do Aquário Carioca geraram efeitos positivos durante o tratamento e cuidado a esta clientela. O espaço do Aquário Carioca está configurado de forma a oferecer às crianças, qualquer que seja a idade, atividades estimulantes e divertidas, permitindo distração, calma e até segurança. Autores apontam o impacto positivo da presença da atividade lúdica durante o período de adoecimento e hospitalização de crianças. .,8-9,18 $^{-18}$ Assim, o brincar passa a ser promovido como um espaço terapêutico capaz de possibilitar a continuidade do desenvolvimento infantil, permitindo à criança hospitalizada melhor enfrentamento do momento específico que vivencia. ${ }^{18}$

Na prática diária, percebeu-se que, no Aquário Carioca, com a mudança da estrutura física, houve alterações nas relações estabelecidas, motivando os profissionais a ampliar e humanizar o cuidado no cotidiano. Assim, os mesmos pareciam se sentir mais valorizados e satisfeitos por trabalharem lá, um ambiente diferenciado. Não raro os membros da equipe de enfermagem convidavam e levavam, ao hospital, familiares para conhecer seu setor de trabalho. A equipe de enfermagem demonstrou maior motivação para realização de suas atividades cotidianas, fato que pode interferir positivamente no relacionamento com as crianças e seus familiares. A aproximação entre a tríade (profissionais-crianças-familiares) possibilita a construção de um processo de cuidado enriquecido por melhorar a comunicação, colaboração e compartilhamento entre eles, contribuindo para a construção de uma ética de coimplicação na produção dos saberes, das práticas e das relações. ${ }^{4}$

$\mathrm{O}$ ambiente com distribuição adequada do espaço físico, objetos, sons e imagens disponíveis, exerce influência sobre as pessoas, sendo importante considerá-lo na realização das práticas do cuidar. Constitui-se aspecto importante do cuidado possibilitar a descodificação dos simbolismos do ambiente hospitalar pelos profissionais de saúde, de forma a adaptar um espaço favorável à ação de cuidados. ${ }^{19}$

O Aquário Carioca contribuiu para o aumento da autoestima da equipe multiprofissional, facilitando os processos de trabalho e a interação junto às crianças e seus familiares, fato relevante para a construção de uma ecologia hospitalar positiva. Com um ambiente amplo, foi possível incorporar diferentes projetos de humanização para trazer o lúdico ao local de tratamento das crianças. Assim, o que antes acontecia em ambientes diferentes hoje pode acontecer em um único lugar, descodificando a ideia da sala onde só se tem sofrimento. Portanto, a resistência a alguns procedimentos invasivos, como manipulação de cateter venoso central, foi minimizada com a utilização, pelas crianças, das tecnologias de distração disponíveis, a inquietação pela demora da infusão foi deixada de lado e o desejo de permanecer na sala mesmo após término do tratamento era constante, influenciando diretamente na qualidade da assistência prestada.

Ademais, com as mudanças na estrutura física, nas relações, e com o uso de estratégias lúdicas, o cuidado pode ser humanizado, na medida em que minimizou a tensão característica do ambiente hospitalar e melhorou as relações interpessoais, viabilizando a interação terapêutica e dialógica entre crianças, equipe e família.

\section{CONCLUSÃO}

Os diferentes aspectos ${ }^{9}$ que estão envolvidos com a ecologia hospitalar precisam ser mais valorizados pela enfermagem, já que exerce influência sobre as pessoas que ali convivem refle- 
tindo no desenvolvimento do cuidado. A criança interage com o ambiente onde se encontra e esta relação pode interferir diretamente no processo saúde/doença, por isso, a humanização deve ser considerada prioridade em serviços de atenção pediátrica.

O Aquário Carioca é hoje referência do processo de humanização da assistência desta Instituição. Como esta ainda não é a realidade dos outros setores torna-se necessário a expansão de ambientes como este e aumentar o número de crianças que se beneficiam com a utilização do Aquário Carioca, tendo em vista que o setor é de uso exclusivo das crianças em quimioterapia antineoplásica ambulatorial.

No entanto, é fundamental a realização de estudos sobre a influência do ambiente e seus benefícios para a saúde das crianças e o cuidado de enfermagem. Recomenda-se estudar e verificar a relação com a diminuição dos efeitos colaterais do tratamento, bem como outros sinais e sintomas das crianças usuárias de tais ambientes.

\section{REFERÊNCIAS}

1. Menezes CNB, Passareli PM, Drude FS, Santos MA, Valle ERM. Câncer infantil: organização familiar e doença. Rev Mal-Estar Subj. 2007 Mar; 7(1):191-210.

2. Araújo YB, Collet N, Moura FM, Nóbrega RD. Conhecimento da família acerca da condição crônica na infância. Texto Contexto Enferm. 2009 Set; 18(3):498-505.

3. Gomes IP, Reis PED, Collet N. Gerenciando o cuidado de enfermagem no ambulatório de quimioterapia pediátrica: relato de experiência. Rev Enferm UFPE on line [online]. 2010[acesso 2010 abr 20]; 4(2). Disponível em: http:/ / www.ufpe.br/ revistaenfermagem

4. Souza LA de P, Mendes VLF. O conceito de humanização na Política Nacional de Humanização $(\mathrm{PNH})$. Interface Comun Saúde Educ [online]. 2009[acesso em 2010 jan 04]; 13(suppl. 1). Disponível em: http:/ / www.scielo.br/pdf/icse/v13s1/ a18v13s1.pdf

5. Guelli A, Zucchi P. A influência do espaço físico na recuperação dos pacientes e os sistemas e instrumentos de avaliação. Rev Adm Saúde. 2005 Abr; 7(27):43-50.

6. Cavalcanti PB, Azevedo GAN, Duarte CR. Humanização, imagem e caráter dos espaços de saúde. Cadernos Proarq. 2007; 11:7-10.

7. Ministério da Saúde (BR). Secretaria de Atenção à Saúde. Portaria N²81, de 19 de junho de 2001: Institui o Programa nacional de humanização da assis- tência hospitalar (PNHAH). Brasília (DF): MS, 2001.

8. Morsch SS, Aragão PM. A criança, sua família e o hospital: pensando processos de humanização. In: Deslandes SF (organizadora). Humanização dos cuidados em saúde: conceitos, dilemas e práticas. Rio de Janeiro (RJ): Fio Cruz; 2008. p.235-260.

9. Gomes IP. Influência do ambiente na percepção das crianças em quimioterapia ambulatorial [dissertação]. João Pessoa (PB): Universidade Federal da Paraíba, Programa de Pós-Graduação em Enfermagem; 2011.

10. Dyniewicz, AM. Metodologia. In: Metodologia da pesquisa em saúde para iniciantes. São Caetano do Sul (SP): Difusão, 2007. p.105-28.

11. Ministério da Saúde (BR), Conselho Nacional de Saúde, Comissão Nacional de Ética em Pesquisa. Resolução $N^{\circ} 196$ de 10 de outubro de 1996: diretrizes e normas regulamentadoras de pesquisa envolvendo seres humanos. Brasília (DF): MS; 1996.

12. Moreira MCN, Mitre RMA. Instituto Desiderata [página da internet]. A humanização das salas de quimioterapia pediátricas do Rio de Janeiro: o hospital pelo olhar das crianças. [acesso 2009 Out 5]. Disponível em: http://www.desiderata.org.br/ docs/relatorio_humanizacao.pdf

13. Instituto Desiderata. [página da internet]. Oncologia pediátrica: programa ampliando o acolher. [acesso 2009 Out 5]. Disponível em: http:/ / www.desiderata. org.br/

14. Bonassa EMA. Efeitos colaterais dos antineoplasicos. In: Bonassa EMA, Santana TR. Enfermagem em terapêutica oncológica. São Paulo (SP): Atheneu; 2005, p.101-30.

15. Silva D, Reis PED, Gomes IP, Funghetto S, Poncede-Leon C. Non pharmacological interventions for chemotherapy induced nauseas and vomits: integrative review. Online Braz J Nurs. [online]. 2009 Mar; [acesso 2010 Abr 20]; 8(1). Disponível em: http:/ / www.objnursing.uff.br/index.php/ nursing/article/view/2098

16. Ferreira CCM, Remedi PP, Lima RAG. A música como recurso no cuidado à criança hospitalizada: uma intervenção possível?. Rev Bras Enferm [online]. 2006 Out [acesso 2010 Abr 20]; 59(5):689-93. Disponível em: http:/ / www.scielo.br/pdf/reben/ v59n5/v59n5a18.pdf

17. Abreu MAV, Reis PED, Gomes IP, Rocha PRS. Non pharmacologic pain management on oncologic patients: systematic review. Online Braz J Nurs. [online]. 2009 Mar; [acesso 2010 May 9]; 8(1). Disponível em: http://www. objnursing.uff.br/index.php/nursing/article/vie w/j.1676-4285.2009.2222/47516.

18. Mitre RMA, Gomes R. A promoção do brincar no contexto da hospitalização infantil como ação de 
saúde. Ciênc Saúde Coletiva, [online] 2004 [citado 2009 Out 27]; 9(1). Disponível em: http:/ /www. scielo.br/pdf/csc/v9n1/19832.pdf
19. Collière MF. Promover a vida: da prática das mulheres de virtude aos cuidados de enfermagem. Lisboa (PT): Sindicato dos Enfermeiros Portugueses; 1999.

E-mail: enfisabelle@yahoo.com.br 